\title{
Expression of Smad1 is directly associated with mesangial matrix expansion in rat diabetic nephropathy
}

\author{
Takeshi Matsubara ${ }^{1}$, Hideharu Abe ${ }^{2}$, Hidenori Arai ${ }^{3}$, Kojiro Nagai ${ }^{1}$, Akira Mima ${ }^{1}$, \\ Hiroshi Kanamori ${ }^{1}$, Eriko Sumi ${ }^{1}$, Toshikazu Takahashi ${ }^{2}$, Motokazu Matsuura ${ }^{2}$, \\ Noriyuki Iehara ${ }^{1}$, Atsushi Fukatsu ${ }^{1}$, Toru Kita ${ }^{4}$ and Toshio Doi $^{2}$ \\ ${ }^{1}$ Department of Nephrology, Kyoto University Graduate School of Medicine, Kyoto, Japan; ${ }^{2}$ Department of \\ Clinical Biology and Medicine, Tokushima University School of Medicine, Tokushima, Japan; ${ }^{3}$ Department \\ of Geriatric Medicine, Kyoto University Graduate School of Medicine, Kyoto, Japan and ${ }^{4}$ Department of \\ Cardiovascular Medicine, Kyoto University Graduate School of Medicine, Kyoto, Japan
}

\begin{abstract}
Diabetic nephropathy is the leading cause of end-stage renal disease, and glomerular mesangial matrix expansion is the hallmark in diabetic nephropathy. However, the precise mechanism for the development of mesangial matrix expansion has remained unknown. The key component involved in mesangial matrix expansion is type IV collagen (Col4). Recently, we have reported that Smad1 transcriptionally regulates expression of Col4 under diabetic conditions in vitro. Here we show that this direct regulator of Col4 also plays a crucial role for mesangial matrix expansion in vivo. Streptozotocin-induced diabetic rats are the model of incipient diabetic nephropathy, and showed various levels of mesangial matrix expansion at 24 weeks. The glomerular expression of Smad1 was significantly increased in diabetic rats with more mesangial matrix expansion by Western blot and immunohistochemical analysis. Furthermore, the glomerular expression of Smad1 was closely correlated with the glomerular expression of Col4 and smooth muscle alpha actin ( $\alpha$-SMA), while albuminuria or glomerular filtration rate was not correlated with mesangial matrix expansion. We also found that urinary excretion of Smad1 was closely associated with the severity of mesangial matrix expansion. In cultured mesangial cells expression of Smad1 upregulated the transcriptional activity of key molecules in mesangial matrix expansion, such as Col4 and $\alpha$-SMA. These data indicate the critical involvement of Smad1 in mesangial matrix expansion in the early phase of diabetic nephropathy. Our data imply that urinary Smad1 might be a representative diagnostic marker for mesangial matrix expansion in diabetic nephropathy. Laboratory Investigation (2006) 86, 357-368. doi:10.1038/labinvest.3700400; published online 13 February 2006
\end{abstract}

Keywords: diabetic nephropathy; mesangial matrix expansion; Smad1; smooth muscle alpha actin; type IV collagen

Diabetic nephropathy is the leading cause of endstage renal disease in the United States, Japan, and so on. The major morphological changes are characterized by thickening of glomerular basement membrane (GBM) and mesangial matrix expansion. The latter is more important, because there is widespread agreement that they are generally accompanied by various tubulointerstitial damages followed by nephron loss to end-stage renal failure. ${ }^{1,2}$ The

Correspondence: Dr H Arai, MD, PhD, Department of Geriatric Medicine, Kyoto University Graduate School of Medicine, 54 Kawahara-cho, Shogoin, Sakyo-ku, Kyoto 606-8507, Japan.

E-mail: harai@kuhp.kyoto-u.ac.jp

Received 18 September 2005; revised and accepted 13 January 2006; published online 13 February 2006 decline of glomerular filtration rate (GFR) is associated with the increasing number of obsolescent glomeruli $^{3}$ and the severity of mesangial matrix expansion in type I diabetes. On the other hand, the thickening of GBM has little or no correlation with the decline of GFR. ${ }^{4}$ Therefore, mesangial matrix expansion is the hallmark from the point of pathological and clinical events in diabetic nephropathy. Glomerulosclerosis is defined as the segmental or global collapse or closure of capillary loops with associated mesangial matrix expansion. However, the early stage of diabetic nephropathy is associated with glomerular hyperfiltration and glomerular hypertrophy, but not collapse of glomerular capillaries. Therefore, mesangial matrix expansion is one of the most important pathological findings in the early phase of diabetic nephropathy. 
Albuminuria is one of the most characteristic functional changes in the early phase of diabetic nephropathy. Recent study suggests that the percentage of microalbuminuric patients progressing to proteinuria over $\sim 10$ years is only $30-45 \% .^{5}$ Furthermore, extensive studies of the glomerular structure in diabetic patients with or without microalbuminuria failed to find a significant difference in the glomerular structural changes such as mesangial matrix expansion between the two groups in the absence of a raised blood pressure or reduced creatinine (Cre) clearance. ${ }^{6,7}$ These reports show that albuminuria might not be a definite marker for mesangial matrix expansion in the early phase of diabetic nephropathy, although albuminuria correlates with and predicts the late development of diabetic nephropathy with decreased GFR and glomerulosclerosis.

Mesangial matrix expansion is characterized by increased amounts of extracellular matrix (ECM), including alpha 1/alpha 2 type IV collagen (Col4), laminin, type I and III collagens, heparan sulfate proteoglycan, and fibronectin. ${ }^{8}$ One of the key components in the pathophysiology is Col4. Various peptides of growth factors are shown to mediate the regulation of this key component. ${ }^{9}$ However, the direct regulation of Col4 had not been proved until our recent report that Smad1 can directly bind to the promoter of Col4, and can upregulate its transcriptional activity. ${ }^{10}$ We also found that advanced glycation endproducts (AGE) can induce the phosphorylation of Smad1 in cultured mesangial cells, and that Smad1 is highly expressed in human advanced diabetic nephropathy. ${ }^{10}$ Thus, Smad1 is the first molecule shown to regulate the expression of Col4 directly under diabetic condition in vitro. However, these findings are obtained only in cultured mesangial cells, and the precise mechanism for the development of mesangial matrix expansion in vivo remains unknown. Therefore, the aim of this study is to investigate the role of Smad1 in diabetic nephropathy in vivo using experimental diabetic animals.

The streptozotocin-induced diabetic (STZ) rat, a type I diabetic model, is known to have glomerular functional changes including albuminuria and the increasing of GFR as seen in the early phase of diabetic nephropathy. ${ }^{11}$ However, STZ rats show only minimal glomerular structural changes even after 1 year of diabetes. ${ }^{12}$ We have induced various levels of mesangial matrix expansion in this model at 24 weeks by feeding special breeding chows. The glomerular structural characteristics in these rats were similar to human early glomerular structural changes, which are a useful model for analyzing mesangial matrix expansion in the early phase of diabetic nephropathy.

In this study, we have examined the role of Smad1 for the development of mesangial matrix expansion in vivo, and also studied whether urinary excretion of Smad1 could be a new representative maker for detecting severity of mesangial matrix expansion. In vitro, we examined whether Smad1 can regulate the transcriptional activity of Col4 and smooth muscle alpha actin ( $\alpha$-SMA) in mesangial cells.

\section{Materials and methods}

\section{Animals and Cell Culture}

Male Sprague-Dawley rats were purchased from Shimizu Laboratory Animal center (Hamamatsu, Japan). All of animal experiments were performed in accordance with institutional guidelines, and the Review Board of Kyoto University granted ethical permission to this study. The glomerular mesangial primary culture was established and maintained according to the method previously described. ${ }^{13}$

Male rats weighing 170-200 g were made diabetic by a single intravenous injection of STZ (Wako, Osaka, Japan) $(55 \mathrm{mg} / \mathrm{kg}$ body weight (BW)) in $0.05 \mathrm{~mol} / \mathrm{l}$ citrate buffer ( $\mathrm{pH} 4.5$ ). Rats receiving an injection of citrate buffer were used as controls. The levels of blood glucose were determined 2 days after injection of STZ or vehicle, and rats with blood glucose levels more than $>16.7 \mathrm{mmol} / \mathrm{l}$ were used. To develop various severities of mesangial matrix expansion in these rats, we used special chows suitable for breeding, containing slightly more protein, and less fat. (LABO H STANDARD, Nosan Corporation, Yokohama, Japan) At 24 weeks after STZ injection, the rats were weighted and killed.

\section{Renal Histology and Morphometric Analyses}

Renal tissue blocks were fixed in ethyl Carnoy's solution, and embedded in paraffin. Sections $(2 \mu \mathrm{m})$ were stained with periodic acid-Schiff methenamine (PASM). To study the severity of mesangial matrix expansion, we quantified mesangial matrix area by measuring mesangial PASM-positive area using an image analyzer with a microscope (Image Processor of Analytical Pathology; IPAP: Sumitomo Chemical Co., Tokyo, Japan). ${ }^{14,15}$ The mesangial matrix fraction (Mes Fx) was determined as percentage of mesangial PASM-positive area per total glomerular surface area. For each animal, 50 glomeruli were analyzed.

\section{Immunohistochemistry}

Kidney sections were processed for immunohistochemistry following standard procedures. Ethyl Carnoy's solution-fixed and paraffin-embedded tissue blocks were used. Kidney sections were rehydrated and treated with $0.3 \% \mathrm{H}_{2} \mathrm{O}_{2}$ in methanol for $30 \mathrm{~min}$. Sections were blocked with the appropriate preimmune serum, and then incubated with AvidinD and Biotin blocking solutions (Vector, CA, USA). Sections were incubated with the anti-Col4 antibody (PROGEN, Heidelberg, Germany), and 
anti-Smad1 antibody (Santa Cruz, CA, USA) overnight at $4{ }^{\circ} \mathrm{C}$ and then incubated with the appropriate biotinylated secondary antibodies followed by incubation with the avidin-biotin peroxidase complex (Vector). Peroxidase conjugates were subsequently localized using diamino-benzidine. To study $\alpha$-SMA and AGE, the tissues were snap frozen in OCT compound (Sakura Finetechnical, Tokyo, Japan), and then cut in $4 \mu \mathrm{m}$-thick sections and fixed in acetone for $10 \mathrm{~min}$, and treated with $0.3 \% \mathrm{H}_{2} \mathrm{O}_{2}$ in methanol for $30 \mathrm{~min}$. Sections were treated with anti- $\alpha$-SMA antibody (clone 1A4, SIGMA), and antiAGE antibody (clone 6D12, Transgenic Co. Ltd., Kumamoto, Japan). Sections were counterstained with hematoxylin solution.

\section{Immunofluorescence Staining and Morphometric Analyses of Glomerular Col4 Expression}

Immunofluorescence staining for Col4 was performed using frozen kidney sections. Sections $(4 \mu \mathrm{m})$ were fixed in acetone, blocked with $10 \%$ donkey serum, and incubated with anti-Col4 antibody followed by incubation with FITC labeled secondary antibody. The immunoreactivity of Col4 was quantified by measuring Col4-positive area in glomeruli using Image-Pro PLUS (Media Cybernetics). The glomerular expression of Col4 was determined as percentage of Col4-positive area per total glomerular surface area. For each animal, 50 glomeruli were analyzed.

\section{Isolation of Glomeruli}

Glomeruli were isolated from renal cortices of rats using the differential sieving method. ${ }^{16,17}$

\section{Western Blotting}

Isolated glomeruli were suspended in RIPA buffer (50 mM Tris, $\mathrm{pH}$ 7.5, $150 \mathrm{mM} \mathrm{NaCl,} \mathrm{1 \%} \mathrm{Nonidet}$ P-40, 0.25\% SDS, $1 \mathrm{mM} \mathrm{Na}_{3} \mathrm{VO}_{4}, 2 \mathrm{mM}$ EDTA, $1 \mathrm{mM}$ phenylmethylsulfonyl fluoride, $10 \mathrm{mg} / \mathrm{ml}$ of aprotinin) for $1 \mathrm{~h}$ at $4^{\circ} \mathrm{C}$. After centrifugation, the supernatants were used as total glomerular lysates. Of each sample ( $40 \mu \mathrm{g}$ ) was applied to SDS-PAGE. After electrophoresis, the proteins were transferred to nitrocellulose filters (Schleicher \& Schuell). The blots were subsequently incubated with rabbit antiSmad1 (Upstate Biotechnology), anti-phospho Smad1/5/8 (Cell Signaling Technology), ALK-1 (Santa Cruz), TGF- $\beta$ (Santa Cruz), and $\alpha$-SMA antibody, following by incubation with horseradish peroxidase-conjugated secondary antibody (Amersham Biosciences). The immunoreactive bands were visualized using the enhanced chemiluminescent system (Amersham Biosciences). Quantitative analysis of the band density was performed using Image Gauge (Fuji Photo Film Co., Ltd).

\section{AGE Preparation}

AGE-bovine serum albumin (BSA) was prepared by the regular methods as described previously. ${ }^{10}$ Unmodified BSA was incubated under the same conditions without glucose 6-phosphate for control. Protein concentration was determined by the Bradford method using BSA as a standard. All AGEprotein-specific fluorescence determinations were performed by measuring emission at $440 \mathrm{~nm}$ upon excitation at $370 \mathrm{~nm}$ using a fluorescence spectrometer (Hitachi). AGE content was estimated by fluorescence intensity at a protein concentration of $1 \mathrm{mg} / \mathrm{ml}$. AGE-BSA contained 89.9 AGE units per mg of protein, and unmodified BSA contained 3.71 AGE units per mg of protein.

\section{Dot Blot Immunoassay}

Dot blots were performed by applying $5 \mu \mathrm{g}$ of glomerular lysates onto a nitrocellulose transfer membrane (PROTRAN). Standard solutions of AGE prepared at concentrations ranging from 0.5 to 32 arbitrary units $/ 5 \mu \mathrm{g}$ total protein with the dilution buffer, were also applied to the membrane, which was then blocked with $5 \%$ skim milk for $1 \mathrm{~h}$. The membrane was then incubated with anti-AGE monoclonal antibody followed by incubation with horseradish peroxidase-conjugated secondary antibody (Amersham Biosciences). The immunoreactive dots were visualized and quantitatively analyzed as described in Western blotting and ELISA procedure.

\section{Blood and Urine Examination}

Blood was collected at killing. Serum Cre, urinary Cre, and blood urea nitrogen were determined with a Hitachi Model 736 autoanalyzer. ${ }^{18} \mathrm{HbA1c}$ was measured using DCA 2000 analyzer (Bayer Medical, Tokyo, Japan). Urine volume was measured at 24 weeks by $24 \mathrm{~h}$ urine collection from rats housed in individual metabolic cages. The Cre clearance was calculated and normalized to BW. The urinary albumin concentration was measured by Nephrat (Exocell Inc.), and adjusted by urinary Cre concentration.

\section{Urine Preparation}

Urine samples were centrifuged for $15 \mathrm{~min}$. The supernatants were stored at $-80^{\circ} \mathrm{C}$ and rapidly thawed and centrifuged to remove any urates or phosphates before use in assays. Because Smad1 concentrations in urine are below the detection limits of our assays, the following methods for concentration were developed and tested.

A 2-ml urine sample was placed in a Centricon-10 filter (Amicon) pretreated with $0.1 \%$ Tween-20 used to limit adsorption to its polypropylene components. The extent of concentration achieved was 
calculated from the exact retenate volume measured with a Hamilton pipet. This ranged from 25- to 60fold, with a median of 55 -fold concentration. This prepared urine concentrate was diluted to total volume of $110 \mu \mathrm{l}$ with the dilution buffer as described below. Subsequently, $100 \mu \mathrm{l}$ of the $110 \mu \mathrm{l}$ total volumes was utilized in the following assays. The final urine concentration was 18 -fold higher than the original sample. This was taken into consideration in the final calculation of the concentration of Smad1 in each urine sample.

\section{ELISA Procedure}

The following buffers were prepared with deionized water and used in ELISA. Coating buffer: $0.1 \mathrm{~mol} / \mathrm{l}$ phosphate buffer, $\mathrm{pH} 7.4$ (PB) containing 3\% BSA (SIGMA). Dilution buffer: PB containing 3\% BSA and $0.1 \%$ Tween-20. Washing buffer: $\mathrm{PB}$ containing $0.1 \%$ Tween-20.

All the ELISA assays were performed in 96-well microtiter plates (Nunc). Anti-Smad1 monoclonal antibody (Santa Cruz) was diluted with the coating buffer to obtain a final concentration of $2.5 \mu \mathrm{g} / \mathrm{ml}$. Aliquots $(250 \mathrm{ng} / 100 \mu \mathrm{l})$ were added to each well, and the plate was incubated overnight at $4^{\circ} \mathrm{C}$. The wells were washed three times. In all $50 \mu \mathrm{l}$ of the prepared urine samples and standard solutions of recombinant Smad1 prepared at concentrations ranging from 0.125 to $512 \mathrm{ng} / \mathrm{ml}$ with the dilution buffer were added to the plate and incubated overnight at $4^{\circ} \mathrm{C}$. The plates were then washed three times. Of rabbit anti-Smad1 polyclonal antibody (Upstate), $100 \mu \mathrm{l}$ was diluted with the diluting buffer to obtain a final concentration of $2.5 \mu \mathrm{g} / \mathrm{ml}$, added to each well, and incubated overnight at $4^{\circ} \mathrm{C}$. Horseradish peroxidase-conjugated anti-rabbit IgG polyclonal antibody (Amersham) was diluted 500-fold with the dilution buffer, and $50 \mu \mathrm{l}$ were then dispensed into each well and the plate was further incubated at room temperature for $120 \mathrm{~min}$. The plates were washed three times.

Of freshly prepared peroxidase substrate solution (DAKO), $50 \mu \mathrm{l}$ were added to each well. The plates were kept at room temperature in the dark for $30 \mathrm{~min}$, and the enzyme reaction was then stopped by adding $50 \mu \mathrm{l}$ of $2 \mathrm{~N} \mathrm{H}_{2} \mathrm{SO}_{4}$ to each well. The optical density was measured at $492 \mathrm{~nm}$ using a microplate reader SPECTRA (TECAN, Austria). Each Smad1 determination was carried out in duplicate. Delta SOFT3 ${ }^{\mathrm{TM}}$ (Biometallics) was used to obtain the calibration curve and the polynomial equation. The optical densities of all urine samples were then inserted in the equation in order to calculate the corresponding Smad1 concentration.

\section{Plasmid Constructs}

The wild-type Col4 promoter reporter plasmid (Col4-LacZ) was constructed as follows: A $0.8 \mathrm{~Kb}$
Srfl-XbaI fragment of the $5^{\prime}$ flanking region of Col4 $\alpha 2$ gene was subcloned into the $\beta$-gal Reporter Vector, pNASS $\beta$ (Clontech). The $\alpha$-SMA promoter reporter plasmid (SMA-Luc) contains $1074 \mathrm{bp}$ of the proximal $5^{\prime}$-flanking region plus $43 \mathrm{bp} 5^{\prime}$-untranslated region of the $\alpha$-SMA gene ${ }^{19}$ that is subcloned into the luciferase reporter vector (Promega).

\section{Reporter Assay}

Mesangial cells $\left(1.0 \times 10^{5}\right)$ were seeded into 12 -well plates (Nunc). After $6 \mathrm{~h}$, the cells were transfected with $500 \mathrm{ng}$ of mouse Col4-LacZ reporter construct, or $500 \mathrm{ng}$ of mouse SMA-Luc along with either $500 \mathrm{ng}$ of vector encoding wild type of Smad1 or the mock vector. Transfection was performed with FuGENE6 transfection reagent (Roche) according to the manufacture's instructions. After $48 \mathrm{~h}$, the cells were harvested in reporter lysis buffer. $\beta$-Galactosidase and luciferase activity were measured using the luminescent $\beta$-galactosidase reporter system (BD Bioscience) and the luciferase reporter system from Promega. $\beta$-Galactosidase and luciferase activities were normalized to total protein (RC DC Protein Assay, Bio-Rad). Transfection experiments were quadruplicated, and repeated at least three times. The $\beta$-galactosidase and luciferase activity data were expressed as means \pm s.d.

\section{Statistical Analysis}

Correlation was determined by Spearman's correlation analysis. Regression formulations were obtained by linear regression analysis. As a result of their skewed distribution, glomerular expression of $\alpha$-SMA, and Smad1, and urinary excretion of Smad1 were logarithmically transformed before statistical analysis. All analyses were performed using StatView (SAS Institute, Cary, CA, USA). A $P$-value $<0.05$ was considered statistically significant. All values were expressed as the mean \pm s.d., except for glomerular expression of $\alpha$-SMA, and Smad1, and urinary excretion of Smad1, where geometric means were given, and analyzed by Mann-Whitney nonparametric analysis, or oneway analysis of variance with a modified $t$-test. Statistical significance was defined as $P<0.05$. Quantitation of immunohistochemistry was analyzed by one-way ANOVA followed by the post hoc test. $P$-values $<0.05$ were considered significant. Data are expressed as means \pm s.d.

\section{Results}

\section{STZ-Induced Diabetic Rats Show Various Severities of Mesangial Matrix Expansion}

We first analyzed mesangial matrix expansion in rats after 24 weeks of STZ injection by measuring the Mes Fx (Figure 1). The Mes Fx was significantly 

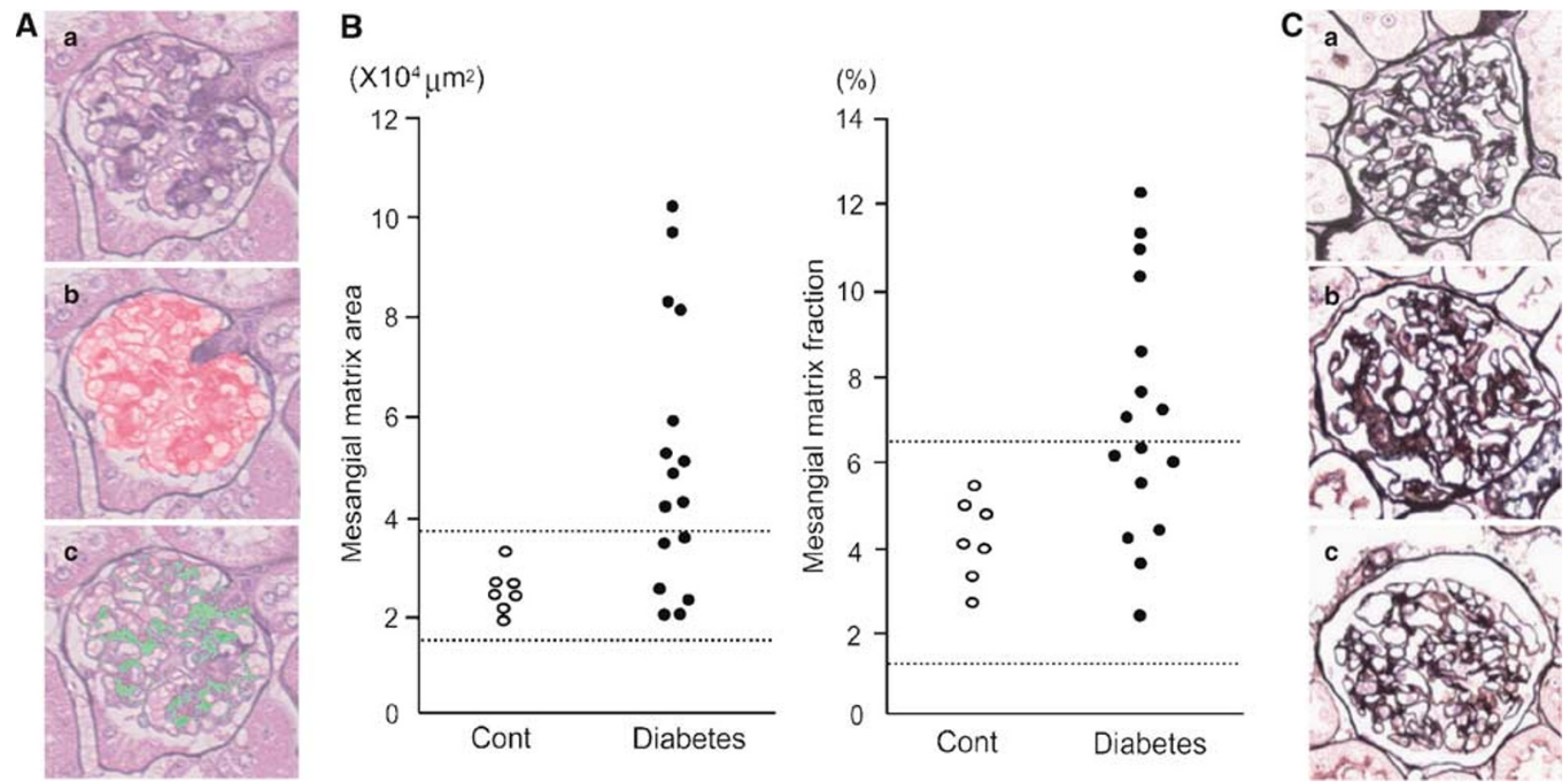

Figure 1 Mesangial matrix fraction in the study groups. (A) Representative glomerulus was shown whose glomerular surface area and mesangial PASM-positive area was determined by IPAP as described in Materials and methods. (a) Image of the PASM stained glomerulus. (b) The assessed glomerular area expressed in red color. (c) Image of the same glomerulus showing the PASM positive area in green color. Note that mesangial PASM-positive area was mainly detected by IPAP. (B) The mesangial matrix fraction was determined as percentage of mesangial matrix area per total glomerular surface area. The distribution of mesangial matrix area (left) and matrix fraction (right) in control and diabetic rats were shown. Each spot represents one rat. The area between two broken lines represents \pm 2 s.d. in control. (C) Representative light microscopic appearance of glomeruli (PASM staining, $\times 400$ original magnification) for control rats (a), diabetic rats with more mesangial matrix expansion (b), and diabetic rats with less mesangial matrix expansion (c). Glomerular hypertrophy and mesangial sclerosis were noted in a glomerulus from a diabetic rat with more mesangial matrix expansion.

increased in diabetic rats $(4.0 \pm 1.1 \%$ in control rats vs $7.0 \pm 2.9 \%$ in diabetic rats, $P<0.05)$. We also noticed that the value of Mes Fx was widely distributed in diabetic rats, and that the values in diabetic rats were overlapped with those in control rats. We then analyzed their physiological characteristics, and examined the relationship between these variables and mesangial matrix expansion. There was no difference in blood pressure between control rats and diabetic rats, or the correlation between blood pressure and the severity of mesangial matrix expansion. HbA1c and blood sugar were increased, and BW was decreased in diabetic rats compared with those in control rats. Serum Cre was decreased in diabetic rats compared with that in control rats (Table 1), reflecting the decrease of BW and the increase of GFR described below. However, all these variables were not correlated with the severity of Mes Fx (data not shown).

\section{Functional Changes do not Correlate with the Glomerular Structural Changes in Diabetic Rats}

In the early phase of diabetic nephropathy, two major functional changes, albuminuria and increased GFR are observed. Therefore, we next mea-
Table 1 Physiological characteristics in this study

\begin{tabular}{lcc}
\hline & Control & Diabetes \\
\hline$N$ & 6 & 16 \\
Body weight (g) & $668 \pm 97$ & $305 \pm 72^{*}$ \\
Blood pressure (mmHg) & $131 \pm 8$ & $136 \pm 6$ \\
Blood glucose (mg/dl) & $112 \pm 7.7$ & $479 \pm 78^{*}$ \\
HbA1c (\%) & $2.8 \pm 0.22$ & $6.6 \pm 1.0^{*}$ \\
Cre (mg/dl) & $0.35 \pm 0.034$ & $0.24 \pm 0.051^{*}$ \\
\hline
\end{tabular}

After 24 weeks of STZ injection, blood pressure (BP) was measured by the cuff-tailed method, and the rats were weighed (BW) and killed. Blood was taken to evaluate blood sugar, HbA1c, and creatinine (Cre). Data were represented as mean \pm s.d.

${ }^{*} P<0.05$ vs control rats.

sured albuminuria and GFR and examined the association of glomerular structural changes with these functional changes. Albuminuria and GFR were significantly increased in both diabetic rats compared with the control rats (control $0.039 \pm$ 0.015 vs diabetes $0.33 \pm 0.14 \mathrm{mg} / \mathrm{mg}$. Cre for albuminuria, and control $8.5 \pm 1.4$ vs diabetes $21 \pm 5.2 \mathrm{ml} /$ day/100 gBW for GFR), but either was not correlated with the severity of mesangial matrix expansion (Figure 2). These data indicate that although albuminuria or GFR could be a sensitive indicator 
of incipient diabetic nephropathy, either of them may not accurately reflect the severity of mesangial matrix expansion in STZ rats.
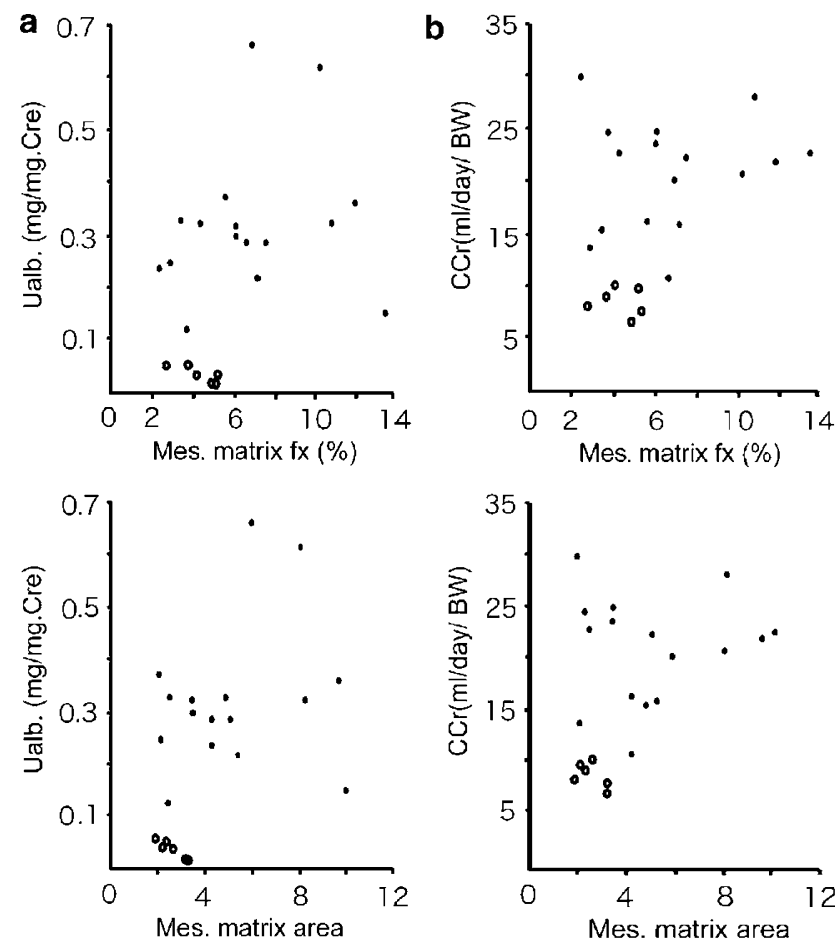

$\left(\mathrm{X} 10^{4} \mu \mathrm{m}^{2}\right)$

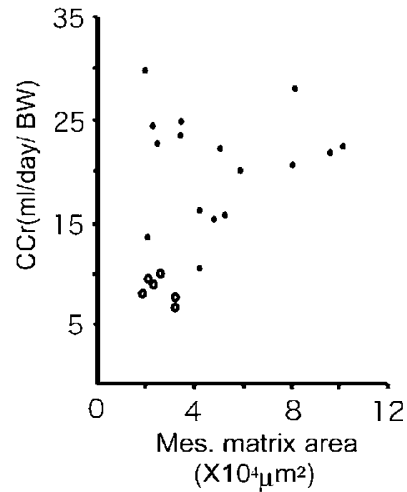

Figure 2 Functional changes in this experiment. The correlations between albuminuria (Ualb; a), or creatinine clearance (CCr; b), and mesangial matrix fraction, or mesangial matrix area were shown. There were no correlations between the two variables and mesangial matrix fraction (upper column) and mesangial matrix area (lower column). Albuminuria was measured by Nephrat, and creatinine clearance was calculated and normalized as described in Materials and methods. Open circles; control rats, filled circles; diabetic rats.

\section{Glomerular Structural Changes are Closely Correlated with the Major Histological Changes in Diabetic Rats}

In diabetic nephropathy, two major histological changes can be found in diabetic glomeruli with mesangial matrix expansion; one is an increase of Col4 in the mesangial area, and the other is an increase of $\alpha$-SMA in mesangial cells. Therefore, to investigate these histological changes in STZ rats, we examined the expression of Col4 (Figure 3) and $\alpha$-SMA (Figure 4) in control and diabetic rats. In diabetic rats, the glomerular expression of Col4 was increased (control $5.8 \pm 1.1 \%$ vs diabetes $8.8 \pm$ $2.9 \%$ ), and was mostly localized in the mesangial area by immunohistochemical analysis. We then examined glomerular expression of $\alpha$-SMA by Western blotting. The glomerular expression of $\alpha$-SMA was almost undetectable in control rats. On the other hand, the glomerular expression of $\alpha$-SMA was significantly increased in diabetic rats with various severities, and its expression was also localized in the mesangial area by immunohistochemical analysis. Furthermore, both histological findings were significantly correlated with the severity of mesangial matrix expansion (Figures $3 \mathrm{~A}$ and $4 \mathrm{~B}$ ).

\section{Glomerular Expression of Smad1 and Phosphorylated Smads is Increased in Diabetic Rats with Greater Mesangial Matrix Expansion}

We previously reported that Smad1 is a direct regulatory factor of Col4 under diabetic conditions in vitro. Therefore, to investigate the role of Smad1 in mesangial matrix expansion in diabetic nephropathy in vivo, we examined the glomerular expression of Smad1 along with phosphorylated Smads by Western blotting and immunohistochemistry (Figure 5). The
A
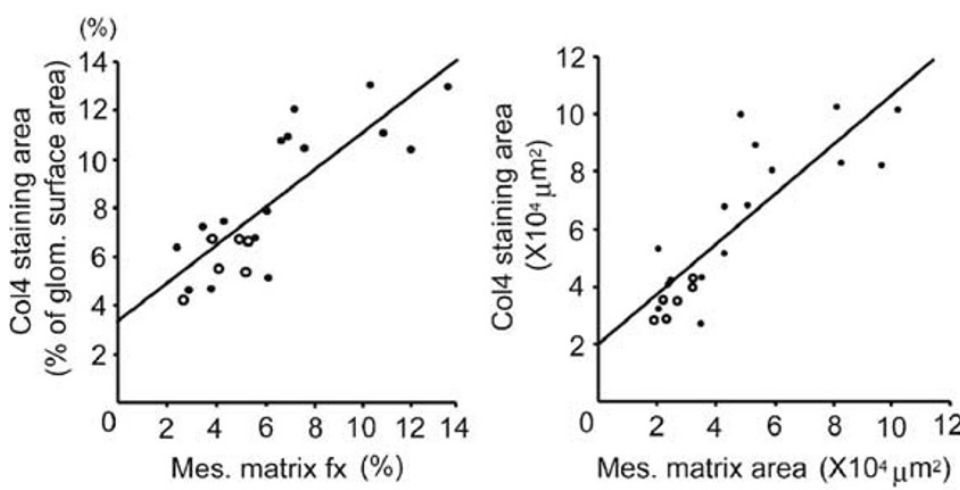

B

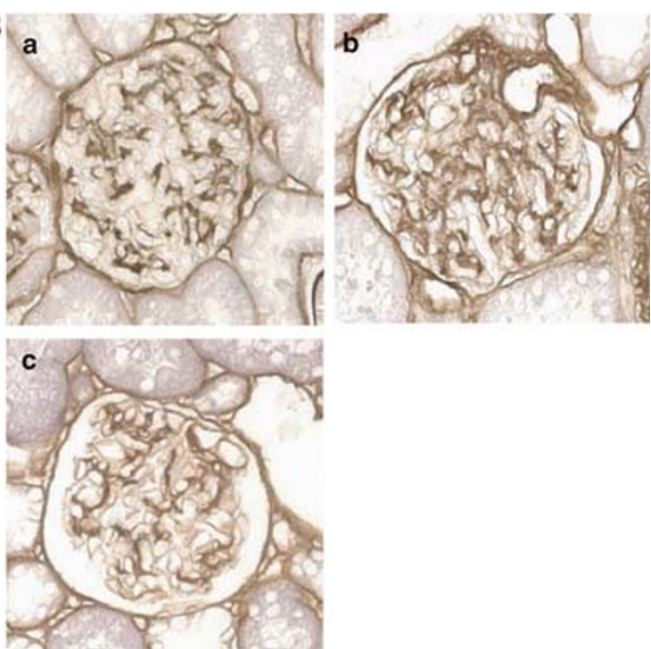

Figure 3 Glomerular expression of Col4. (A) The correlation between glomerular expression of Col4 and mesangial matrix fraction (left), or mesangial matrix area (right) was shown. $r=0.826, P<0.0001$, and $r=0.842, P<0.0001$, respectively. Open circles; control rats, filled circles; diabetic rats. Morphometric analysis of the glomerular expression of Col4 was performed as described in Materials and methods. (B) Immunohistochemistry of Col4 in control rats (a), diabetic rats with more mesangial matrix expansion (b), and diabetic rats with less mesangial matrix expansion (c). The original magnification was $\times 400$. 


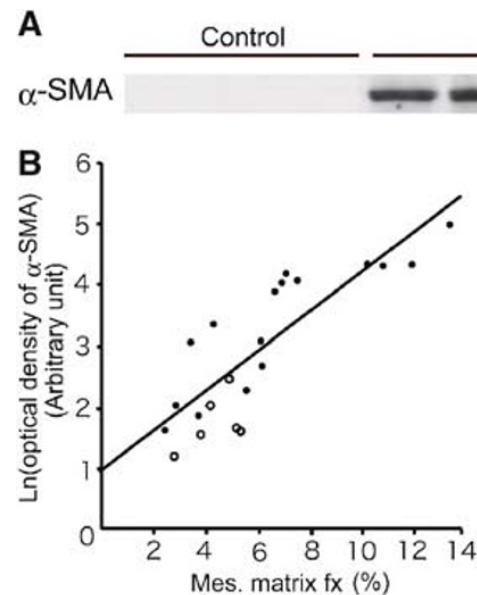

Diabetes

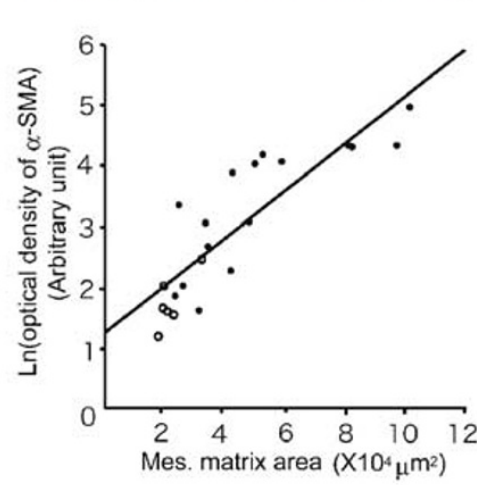

C

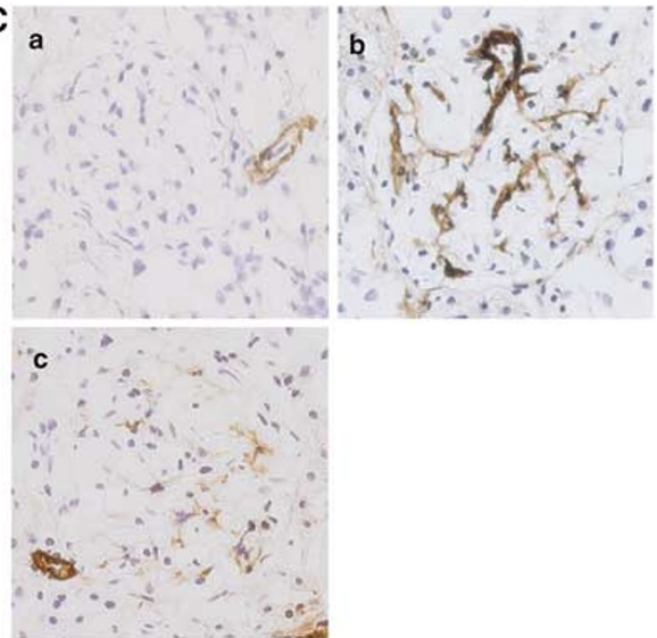

Figure 4 Glomerular expression of $\alpha$-SMA. (A) Immunoblots for glomerular expression of $\alpha$-SMA in control and diabetic rats are shown. Protein $(40 \mu \mathrm{g})$ obtained from each glomerular lysates was loaded. Each lane represents a representative Western blot for the glomerular lysates from each rat. (B) The correlation between glomerular expression of $\alpha$-SMA and mesangial matrix fraction (left) and mesangial matrix area (right) in this study. $r=0.843, P<0.0001$, and $r=0.862, P<0.0001$, respectively. Open circles; control rats, filled circles; diabetic rats. Optical densitometry of $\alpha$-SMA was performed as described. (C) Immunohistochemistry of $\alpha$-SMA in control rats (a), diabetic rats with more mesangial matrix expansion (b), and diabetic rats with less mesangial matrix expansion (c). Sections were counterstained with hematoxylin solution. Representative light microscopic appearance of glomerulus is shown. The original magnification was $\times 400$.

glomerular expression of Smad1 and phosphorylated Smads was significantly increased with distinct varieties, while in control rats, the expression of both Smad1 and phosphorylated Smads was almost undetectable by Western blotting. We also found that increased Smad1 was localized mostly in the glomerular mesangial area. Furthermore, the glomerular expression of Smad1 was strongly correlated with all three major histological findings involved in diabetic glomeruli; the severity of mesangial matrix expansion, the extent of glomerular expression of Col4 and $\alpha$-SMA (Figure 5C). These data strongly suggest that the glomerular expression of Smad1 is closely associated with mesangial matrix expansion in diabetic nephropathy in vivo.

To investigate the factors regulating the glomerular expression of Smad1, we then examined the upstream factors involved in the activation of Smad1. We have already shown that AGE can upregulate the expression and induce the phosphorylation of Smad1 in cultured mesangial cells. ${ }^{10}$ Therefore, first we examined glomerular accumulation of AGE. AGE was detected in expanded mesangial area and GBM in diabetic rats by immunohistochemistry. We then quantified the content of glomerular accumulation of AGE using dot blot analysis of glomerular lysates. A significant correlation was found between glomerular deposition of AGE and glomerular expression of Smad1 $(r=0.494, P<0.05)$. Furthermore, we checked glomerular expression of TGF- $\beta$ and ALK-1, because these molecules are known to act as upstream factors of Smad1, being upregulated by AGE stimulation in cultured mesangial cells. ${ }^{10}$ Compatible with our previous reports, the expression of these molecules was increased in diabetic glomeruli. However, we could not find the correlation between glomerular expression of Smad1 and that of TGF- $\beta$ or ALK-1 (data not shown).

\section{Urinary Excretion of Smad1 is Associated with the Mesangial Matrix Expansion in the Early Phase of Diabetic Nephropathy}

Because the glomerular expression of Smad1 was significantly increased in diabetic rats with mesangial matrix expansion, we examined urinary excretion of Smad1. In our preliminary study, we could detect urinary Smad1 only in some of the urine samples from diabetic rats with mesangial matrix expansion, in proportionate to their glomerular expression of Smad1, by Western blotting. However, the concentration of Smad1 in urine samples was very low, and could not be quantified by Western blotting. Therefore, we developed ELISA to measure the concentration of urinary excretion of Smad1 as described in Materials and methods (Figure 6). In association with an increase of the glomerular expression of Smad1, urinary excretion of Smad1 was increased in diabetic rats by ELISA (control: $0.042 \mathrm{ng} / \mathrm{mg}$. Cre vs diabetic $1.77 \mathrm{ng} /$ $\mathrm{mg} \cdot \mathrm{Cre}$ ). Furthermore, unlike albuminuria or GFR, urinary excretion of Smad1 was closely correlated with Mes Fx, and mesangial matrix area (Figure $6 \mathrm{~b}$ left and right). These data suggest that urinary excretion of Smad1 is also closely associated with mesangial matrix expansion in the early phase of diabetic nephropathy. 
A

Control

Diabetes

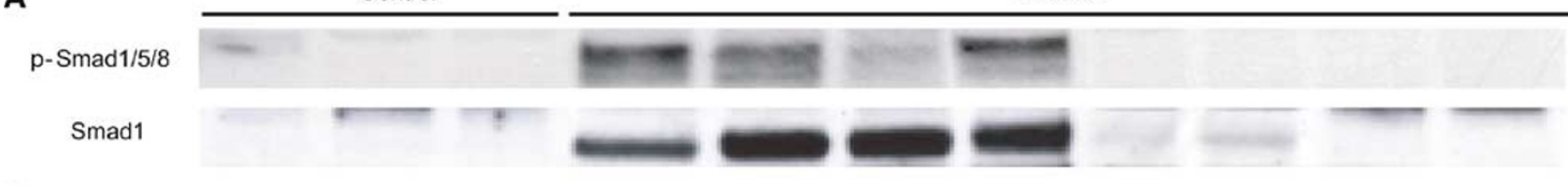

B
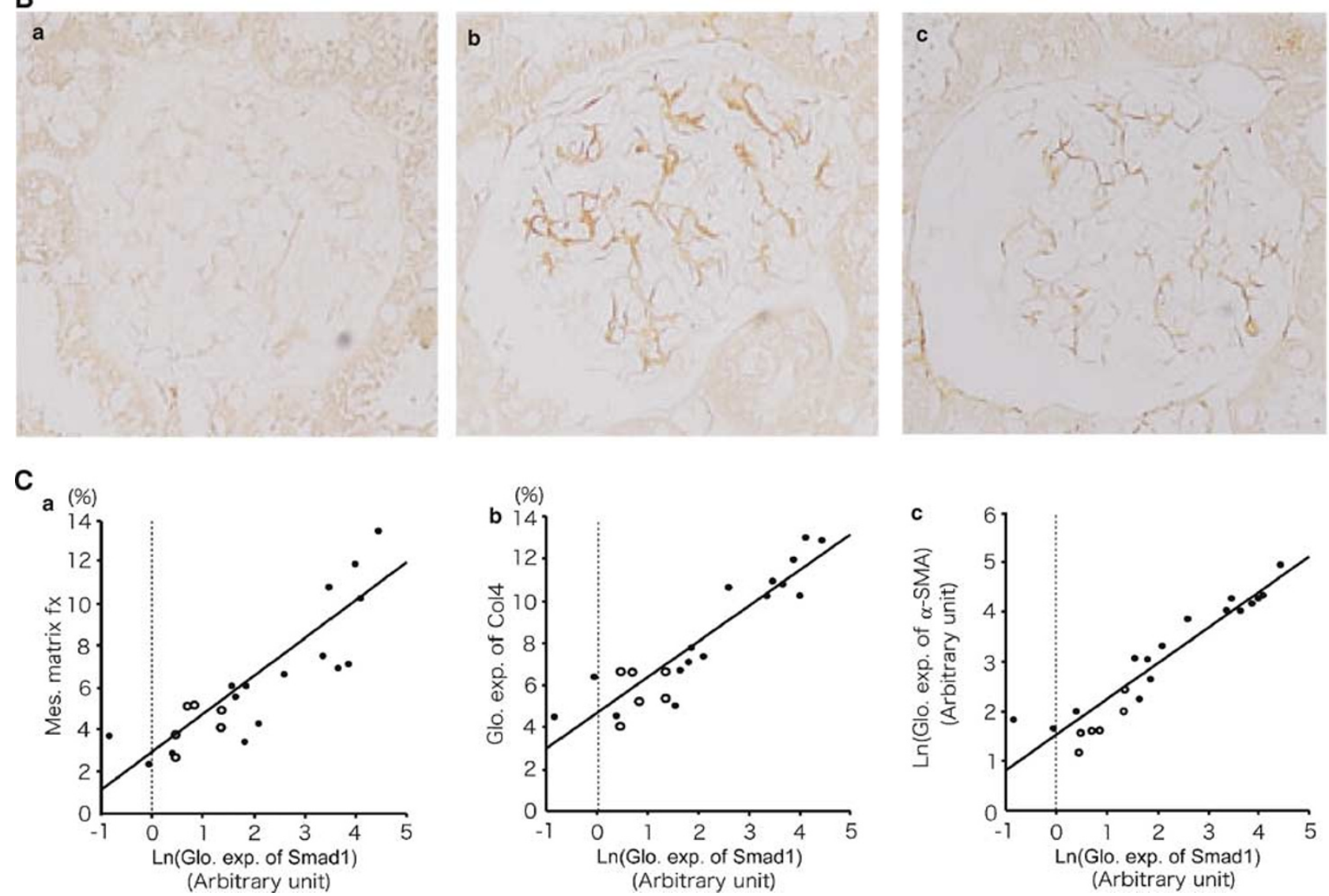

Figure 5 Glomerular expression of Smad1 and phospho-Smads. (A) Immunoblots for Smad1 and phospholylated Smad1/5/8 are shown. Glomerular lysates were prepared as described in Figure 4. Of each sample, ( $40 \mu \mathrm{g})$ was analyzed. Each lane represents a representative Western blot for the glomerular lysates from each rat. (B) Immunohistochemistry of Smad1 in control rats (a), diabetic rats with more mesangial matrix expansion (b), and diabetic rats with less mesangial matrix expansion (c). Representative light microscopic appearance of glomerulus is shown. The original magnification was $\times 400$. (C) The correlation between glomerular expression of Smad1 and mesangial matrix fraction (a; $r=0.850, P<0.0001$ ), glomerular expression of Col4 (b; $r=0.871, P<0.0001$ ), and $\alpha$-SMA (c; $r=0.944$, $P<0.0001)$ in this study. Optical densitometry of glomerular expression of Smad1 in Western blotting was performed as described.

\section{Smad1 is Involved in the Expression not only of Col4, but also of $\alpha$-SMA in Mesangial Cells}

As described above, the glomerular expression of Col4 and $\alpha$-SMA was significantly increased in parallel with the glomerular expression of Smad1. To investigate the relationship among Col4, $\alpha$-SMA, and Smad1, we examined whether Smad1 can cause the increase of Col4 and $\alpha$-SMA expression in mesangial cells in vitro (Figure 7). Transfection of a Smad1 expression vector resulted in an increase in the transcriptional activity of Col4 and $\alpha$-SMA about by two- and four-folds in mouse mesangial cells, respectively. These data suggest that Smad1 is involved in the expression of both Col4 and $\alpha$-SMA in mouse mesangial cells.

\section{Discussion}

Here we demonstrate that Smad1 is a key transcriptional factor by regulating the expression of Col4 and $\alpha$-SMA, and plays an important role for the progression of mesangial matrix expansion in diabetic nephropathy in vivo. Our study also indicates that the amount of urinary Smad1 excretion would be a better diagnostic marker than albuminuria to assess mesangial matrix expansion in diabetic nephropathy.

This study showed the close relationship between mesangial matrix expansion and the glomerular expression of Smad1 in STZ rats. However, we could not show what causes the variety of mesangial matrix expansion and induces glomerular 

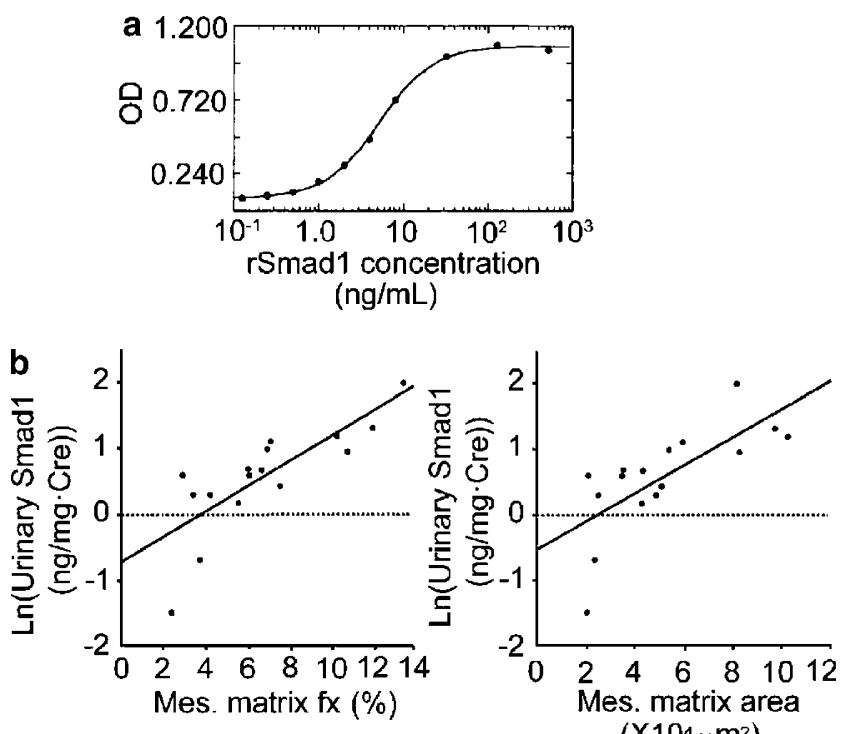

Figure 6 Urinary concentration of Smad1 (a) Standard curve of ELISA. The intra-assay and interassay coefficient variation for this assay were 3.8 and $17.5 \%$, respectively. (b) The correlation between urinary excretion of Smad1 and mesangial matrix fraction (left), or mesangial matrix area (right) in diabetic rats. $r=0.733, P<0.01$, and $r=0.716, P<0.01$, respectively. Quantification of urinary concentration of Smad1 was performed as described in Materials and methods. The variables of control rats were scaled out of the ranges.
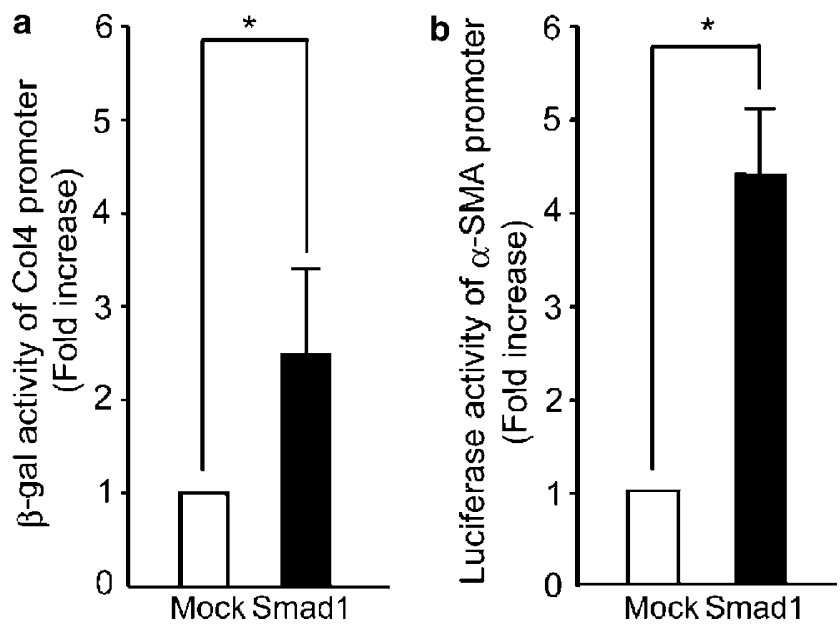

Figure 7 Effects of Smad1 on Col 4 and $\alpha$-SMA transcriptional activities. Mesangial cells were cotransfected with the mouse Col4 promoter-LacZ reporter construct, or the mouse $\alpha$-SMA promoter luciferase reporter construct along with either the vector encoding wild type of Smad1 or the mock vector. After $48 \mathrm{~h}, \beta$-gal activity for Col4 (a) and luciferase activity for $\alpha$-SMA (b) were measured. Both activities were expressed as fold increase over mock vector cotransfection. Data represent the mean \pm s.d. of triplicated samples. ${ }^{*} P<0.05$.

expression of Smad1 in STZ rats. There are many factors that can influence the severity of mesangial matrix expansion in diabetes; duration of diabetes, glycemic control, blood pressure, diets, and so on. In our experiment, however, we could not find any correlation between these variables and the severity of mesangial matrix expansion. According to our data in this and previous reports, the variety of glomerular structural changes could result from the variety of glomerular expression of Smad1. However, there are only a few reports about factors that could regulate the expression of Smad1, although many reports were published about the activation of Smad1. ${ }^{20,21}$ Consistent with our previous report that AGE can induce Smad1 in vitro, here we have shown a positive correlation between AGE and Smad1 in diabetic glomeruli. We also showed that glomerular expression of TGF- $\beta$ and ALK-1 was increased in diabetic rats. However, the direct correlation between glomerular expression of Smad1 and that of TGF- $\beta$ or ALK-1 was not found. These results raised the possibility that TGF- $\beta$ is not sufficient for the induction of glomerulosclerosis, ${ }^{22}$ or that another signaling pathway could activate Smad1, leading to glomerulosclerosis. In this regard, we have already found that angiotensin II can also activate Smad1 via a Src-dependent pathway both in cultured mesangial cells and STZ rats (Mima A, Matsubara T, et al, unpublished observation). Therefore, we need further studies to elucidate the interaction between these two distinct signaling pathways in the development of mesangial matrix expansion in the early phase of diabetic nephropathy.

Genetic factors could also influence the severity of diabetic nephropathy ${ }^{23,24}$ and could regulate the differential induction of Smad1. In this experiment, we used Sprague-Dawley rats, which were known to be one of the common outbred strains, and therefore each rat seemed to have, to some extent, a different genetic background. Thus, the variety of glomerular expression of Smad1 in this experiment could result from the genetic variety of the factors that can affect the expression of Smad1 under diabetic conditions.

We have already shown that Smad1 was a direct transcriptional regulator of Col4 gene in vivo and in vitro. In this study, we have further extended our study and showed that overexpression of Smad1 resulted in an increase of $\alpha$-SMA gene transcription in vitro. Evidence is accumulating which suggests that mesangial cells, during the process of glomerular injuries, including diabetes mellitus, can undergo a phenotypic modulation in which they markedly upregulate their expression of $\alpha$-SMA, in addition to upregulating the expression of Col4..$^{25,26}$ Therefore, our report suggests that two major different events observed in the phenotypic modulation of mesangial cells under diabetic conditions might occur under the regulation of the common transcriptional factor, Smad1. There is no putative direct binding site for Smad1 in the $\alpha$-SMA promoter sequence. ${ }^{10}$ The $\alpha$-SMA promoter has several consensus DNA sequences, which are involved in various transcription factors, some of which are known to interact with, or to be downstream factors of Smad1. Therefore, Smad1 might regulate $\alpha$-SMA 
transcription indirectly via another transcriptional factor. Analysis of the $\alpha$-SMA promoter is now underway to prove this hypothesis.

Although STZ rats were thought to be useful to study the mechanisms of functional changes in the early phase of diabetic nephropathy, they were inappropriate to examine the relationship between functional and structural changes, because glomerular structural changes occurred in STZ rats vary in incidence and severity. ${ }^{12,27}$ In this study, we could examine their variety of glomerular structure clearly showed that there was no association of functional changes with the severity of glomerular structural changes in this model. Furthermore, the validity of this morphometric analysis was supported by our result that the expression of Col 4 and $\alpha$-SMA was significantly correlated with the severity of mesangial matrix expansion. Thus, this morphometric analysis for mesangial PASM-positive area used in this study would be a useful method for studying about mesangial matrix expansion in STZ rats.

Here, we have also found that glomerular injuries occurred in STZ rats can mimic those occurred in the early phase of human diabetic nephropathy, in terms of the association between functional and structural changes. There are several characteristics, but yet unsolved manifestations in the early phase of human diabetic nephropathy. For example, although nearly all patients have glomerular hyperfiltration in this phase, only part of these patients develops diabetic nephropathy. ${ }^{11}$ Next, even if glomerular structural changes occur, the rates of progression often differ from one another and vary greatly among patients. ${ }^{4}$ It is reported that albuminuria does not reflect the severity of glomerular structural changes of diabetic nephropathy. ${ }^{5}$ It is generally accepted that albuminuria predicts the late development of diabetic nephropathy. However, in our study, albuminuria was not correlated with mesangial matrix expansion, which is a predictor for declining of GFR in the late stage. These problems are also found in early human diabetic nephropathy. ${ }^{6,7}$ In this study, we investigated the hyperfiltration stage, which is separated from the late progression stage. We speculate that this argument might come from the difference of the stage in the development of diabetic nephropathy. Therefore, further examination about STZ rats using this morphometric analysis will uncover the mechanism of these manifestations occurred in human early diabetic nephropathy. For this reason, we next checked the correlation of the molecules examined in this study with albuminuria or Cre clearance. We found statistically significant correlation between $\alpha$-SMA and both Cre clearance $(r=0.457, P<0.05)$ and albuminuria $(r=0.597, P<0.05)$. It is very intriguing that glomerular expression of $\alpha$-SMA was significantly correlated with variables of glomerular hemodynamics. The mesangial cells may have several important beneficial functions in the glomeruli, one of which is the modulation of glomer- ular hemodynamics via their contractile properties. ${ }^{28}$ Our results would indicate the association of glomerular hemodynamics with contractile components in diabetic nephropathy. We also found that glomerular expression of $\alpha$-SMA was correlated with albuminuria. However, this finding could be mistakenly deduced from some statistical outliers, although not statistically confirmed in these small sample numbers. Furthermore, evidence is accumulating on the possible pathogenesis of albuminuria dissociated from that of mesangial expansion, ${ }^{29}$ supported by the observation that long-term treatment of $\mathrm{db} / \mathrm{db}$ mice with blocking antibody against TGF- $\beta$ suppressed glomerular mesangial matrix expansion in the absence of significant modulation of albuminuria. ${ }^{30}$ Further examination of STZ rats will elucidate the molecular mechanisms on the relationship between functional and glomerular structural changes.

It is very intriguing that urinary excretion of Smad1 was significantly increased only in STZ rats with diabetic glomerular structural changes. Because diabetic glomerular structural changes are characterized by the accumulation of ECM, the measurement of matrix molecules, such as Col4 in the urine has recently been considered as a possible marker of diabetic glomerular structural changes. However, changes in these markers do not generally increase until the relatively late phase of the disease. At this stage, renal biopsy is the only way to detect the earlier stages of the disease. ${ }^{31}$ Although monitoring urinary TGF- $\beta$ seems attractive, ${ }^{32}$ the highly ubiquitous nature of this cytokine and the existence of multiple circulating forms make it difficult to interpret the results. Another attractive report showed that urinary CTGF was upregulated in STZ rat prior to the development of albuminuria. ${ }^{33} \mathrm{How}-$ ever, it remained to be elucidated whether CTGF could reflect glomerular structural changes especially in the early phase of diabetic nephropathy. In our study, we have confirmed that urinary Smad1 was closely correlated with the severity of mesangial matrix expansion in diabetic rats. Thus, it is suggested that urinary Smad1 could be a better noninvasive diagnostic marker for mesangial matrix expansion in the early phase of diabetic nephropathy.

In summary, this is the first demonstration that Smad1, a direct regulatory factor of Col4 in mesangial cells, also plays a key role for glomerular structural changes in experimental diabetic nephropathy, and that the expression of this key molecule in glomeruli is closely related to the diabetic structural changes. Urinary Smad1 could be a better diagnostic marker for mesangial matrix expansion in the early phase of diabetic nephropathy than albuminuria.

\section{Acknowledgements}

We thank Hideo Uchiyama (Taigenkai Hospital), Ayumi Hosotani, and Maki Watanabe (Kyoto 
University) for excellent technical assistance. This study was supported by Grants-in Aid from the Ministry of Education, Culture, Science, Sports, and Technology of Japan, Center of Excellence grants from the Ministry of Education, Culture, Science, Sports, and Technology of Japan (12CE2006), a research grant for health sciences from the Japanese Ministry of Health, Labor and Welfare, and a grant from Takeda Medical Research Foundation.

\section{References}

1 Striker GE, Schainuck LI, Cutler RE, et al. Structuralfunctional correlations in renal disease. I. A method for assaying and classifying histopathologic changes in renal disease. Hum Pathol 1970;1:615-630.

2 Kriz W, LeHir M. Pathways to nephron loss starting from glomerular diseases-insights from animal models. Kidney Int 2005;67:404-419.

3 Fogo AB. Diabetic nephropathy: it's in the numbers. Kidney Int 2002;61:2274-2275.

4 Mauer SM, Steffes MW, Ellis EN, et al. Structuralfunctional relationships in diabetic nephropathy. J Clin Invest 1984;74:1143-1155.

5 Caramori ML, Fioretto P, Mauer M. Low glomerular filtration rate in normoalbuminuric type 1 diabetic patients: an indicator of more advanced glomerular lesions. Diabetes 2003;52:1036-1040.

6 Chavers BM, Bilous RW, Ellis EN, et al. Glomerular lesions and urinary albumin excretion in type I diabetes without overt proteinuria. N Engl J Med 198; 320:966-970.

7 Fioretto P, Steffes MW, Mauer M. Glomerular structure in nonproteinuric IDDM patients with various levels of albuminuria. Diabetes 1994;43:1358-1364.

8 Striker LJ, Doi T, Elliot S, et al. The contribution of glomerular mesangial cells to progressive glomerulosclerosis. Semin Nephrol 1989;9:318-328.

9 Striker LJ, Peten EP, Elliot SJ, et al. Mesangial cell turnover: effect of heparin and peptide growth factors. Lab Invest 1991;64:446-456.

10 Abe H, Matsubara T, Iehara N, et al. Type IV collagen is transcriptionally regulated by Smad1 under advanced glycation end product (AGE) stimulation. J Biol Chem 2004;279:14201-14206.

11 O’Donnell MP, Kasiske BL, Keane WF. Glomerular hemodynamic and structural alterations in experimental diabetes mellitus. FASEB J 1988;2:2339-2347.

12 Zatz R, Meyer TW, Rennke HG, et al. Predominance of hemodynamic rather than metabolic factors in the pathogenesis of diabetic glomerulopathy. Proc Natl Acad Sci USA 1985;82:5963-5967.

13 Nagai K, Arai H, Yanagita M, et al. Growth arrestspecific gene 6 is involved in glomerular hypertrophy in the early stage of diabetic nephropathy. J Biol Chem 2003;278:18229-18234.

14 Hirata M, Makibayashi K, Katsumata K, et al. 22Oxacalcitriol prevents progressive glomerulosclerosis without adversely affecting calcium and phosphorus metabolism in subtotally nephrectomized rats. Nephrol Dial Transplant 2002;17:2132-2137.

15 Yamamoto Y, Kato I, Doi T, et al. Development and prevention of advanced diabetic nephropathy in RAGE-overexpressing mice. J Clin Invest 2001;108: 261-268.
16 Ziswiler R, Steinmann-Niggli K, Kappeler A, et al. Mycophenolic acid: a new approach to the therapy of experimental mesangial proliferative glomerulonephritis. J Am Soc Nephrol 1998;9:2055-2066.

17 Pippin JW, Qu Q, Meijer L, et al. Direct in vivo inhibition of the nuclear cell cycle cascade in experimental mesangial proliferative glomerulonephritis with Roscovitine, a novel cyclin-dependent kinase antagonist. J Clin Invest 1997;100:2512-2520.

18 Makibayashi K, Tatematsu M, Hirata $\mathrm{M}$, et al. A vitamin D analog ameliorates glomerular injury on rat glomerulonephritis. Am J Pathol 2001;158: 1733-1741.

19 Iehara N, Takeoka H, Yamada Y, et al. Advanced glycation end products modulate transcriptional regulation in mesangial cells. Kidney Int 1996;50: 1166-1172.

20 Zuscik MJ, Baden JF, Wu Q, et al. 5-azacytidine alters TGF-beta and BMP signaling and induces maturation in articular chondrocytes. J Cell Biochem 2004;92: 316-331.

21 Ota T, Fujii M, Sugizaki T, et al. Targets of transcriptional regulation by two distinct type I receptors for transforming growth factor-beta in human umbilical vein endothelial cells. J Cell Physiol 2002;193: 299-318.

22 Yang CW, Striker GE, Chen WY, et al. Differential expression of glomerular extracellular matrix and growth factor mRNA in rapid and slowly progressive glomerulosclerosis: studies in mice transgenic for native or mutated growth hormone. Lab Invest 1997; 76:467-476.

23 Seaquist ER, Goetz FC, Rich S, et al. Familial clustering of diabetic kidney disease. Evidence for genetic susceptibility to diabetic nephropathy. N Engl J Med 1989;320:1161-1165.

24 Fioretto P, Steffes MW, Barbosa J, et al. Is diabetic nephropathy inherited? Studies of glomerular structure in type 1 diabetic sibling pairs. Diabetes 1999; 48:865-869.

25 Takeoka H, Iehara N, Uematsu-Yanagita M, et al. A multifunctional transcription factor (A1p145) regulates the smooth muscle phenotype in mesangial cells. Biochem Biophys Res Commun 1998;252: 290-295.

26 Abe $\mathrm{H}$, Iehara $\mathrm{N}$, Utsunomiya $\mathrm{K}$, et al. A vitamin $\mathrm{D}$ analog regulates mesangial cell smooth muscle phenotypes in a transforming growth factor-beta type II receptor-mediated manner. J Biol Chem 1999;274: 20874-20878.

27 Steffes MW, Brown DM, Basgen JM, et al. Amelioration of mesangial volume and surface alterations following islet transplantation in diabetic rats. Diabetes 1980;29: 509-515.

28 Johnson RJ, Floege J, Yoshimura A, et al. The activated mesangial cell: a glomerular 'myofibroblast'? J Am Soc Nephrol 1992;2:S190-S197.

29 Wendt TM, Tanji N, Guo J, et al. RAGE drives the development of glomerulosclerosis and implicates podocyte activation in the pathogenesis of diabetic nephropathy. Am J Pathol 2003;162:1123-1137.

30 Ziyadeh FN, Hoffman BB, Han DC, et al. Long-term prevention of renal insufficiency, excess matrix gene expression, and glomerular mesangial matrix expansion by treatment with monoclonal antitransforming growth factor-beta antibody in $\mathrm{db} / \mathrm{db}$ diabetic mice. Proc Natl Acad Sci USA 2000;97:8015-8020. 
31 Mauer SM, Chavers BM, Steffes MW. Should there be an expanded role for kidney biopsy in the management of patients with type I diabetes? Am J Kidney Dis 1990; 16:96-100.

32 Ellis D, Forrest KY, Erbey J, et al. Urinary measurement of transforming growth factor-beta and type IV collagen as new markers of renal injury: application in diabetic nephropathy. Clin Chem 1998;44:950-956.

33 Riser BL, Cortes $\mathrm{P}$, DeNichilo $\mathrm{M}$, et al. Urinary CCN2 (CTGF) as a possible predictor of diabetic nephropathy: preliminary report. Kidney Int 2003;64: 451-458. 